\title{
Hepatic function and the cardiometabolic syndrome
}

This article was published in the following Dove Press journal:

Diabetes, Metabolic Syndrome and Obesity:Targets and Therapy

9 October 2013

Number of times this article has been viewed

\section{Nicolas Wiernsperger \\ INSERM French Institute of Health and Medical Research, U1060, National Institute \\ of Applied Sciences, Lyon, University of Lyon, Villeurbanne, France}

\begin{abstract}
Despite skeletal muscle being considered by many as the source of insulin resistance, physiology tells us that the liver is a central and cardinal regulator of glucose homeostasis. This is sometimes underestimated because, in contrast with muscle, investigations of liver function are technically very difficult. Nevertheless, recent experimental and clinical research has demonstrated clearly that, due in part to its anatomic position, the liver is exquisitely sensitive to insulin and other hormonal and neural factors, either by direct intrahepatic mechanisms or indirectly by organ cross-talk with muscle or adipose tissue. Because the liver receives absorbed nutrients, these have a direct impact on liver function, whether via a caloric excess or via the nature of food components (eg, fructose, many lipids, and trans fatty acids). An emerging observation with a possibly great future is the increase in intestinal permeability observed as a consequence of high fat intake or bacterial modifications in microbiota, whereby substances normally not crossing the gut gain access to the liver, where inflammation, oxidative stress, and lipid accumulation leads to fatty liver, a situation observed very early in the development of diabetes. The visceral adipose tissue located nearby is another main source of inflammatory substances and oxidative stress, and also acts on hepatocytes and Kupffer cells, resulting in stimulation of macrophages. Liberation of these substances, in particular triglycerides and inflammation factors, into the circulation leads to ectopic fat deposition and vascular damage. Therefore, the liver is directly involved in the development of the prediabetic cardiometabolic syndrome. Treatments are mainly metformin, and possibly statins and vitamin D. A very promising avenue is treatment of the leaky gut, which appears increasingly to be an important causal factor in hepatic insulin resistance and steatosis.
\end{abstract}

Keywords: nonalcoholic liver disease, fatty liver, insulin resistance, cardiometabolic syndrome, pharmacology

\section{Cardiometabolic syndrome: definitions and limits}

The prediabetic period, which can last for decades, is characterized by metabolic modifications which eventually lead to frank type 2 diabetes. This situation was initially termed "syndrome X", but soon evolved to "metabolic syndrome". With increasing recognition that these metabolic disorders also lead to cardiovascular complications (myocardial infarction, stroke), the terminology was changed further to the notion of "cardiometabolic syndrome". Indeed, the prevalence of large vessel disease appears to be essentially similar to that of already established type 2 diabetes. Microvascular abnormalities were completely ignored for a long time, but more recent investigations have revealed that modifications such as microalbuminuria, retinal blood flow change, and even signs of nonenzymatic glycation, albeit far less pronounced than in
Correspondence: Nicolas Wiernsperger CarMeN Laboratory INSA, Nationa Institute of Applied Sciences, University of Lyon, IMBL, Villeurbanne, F-6962I France

Email remedes@orange.fr 
type 2 diabetes, can be found if screened for before fasting hyperglycemia is present.

Cardiometabolic syndrome is an extremely complicated issue due to the fact that it encompasses a mesh of metabolic pathways (mainly glycemia and lipids) and involves several tissues (liver, fat, muscle, and others). For example, the liver is closely involved not only in regulation of glycemia and lipids but also in inflammation and hemostasis, which are main players (as will be seen) in cardiometabolic syndrome. Moreover, many of these processes operate in a bidirectional manner.

According to the various definitions used to ascertain metabolic syndrome, the prevalence in a "normal" population is around $30 \%$, varying with many parameters, including gender, ethnicity, geographic distribution, standard of living, and smoking. Overweight and obesity are found at some step of the metabolic syndrome in a large majority of subjects, which represents a main bias for interpreting the "mechanistic" evolution of metabolic syndrome and its links with cardiovascular complications.

The "search for the Grail" question therefore is: what comes first? The answer is unknown, and the most likely and reasonable response is that there is no answer. Indeed, several hypotheses have been proposed around the notion of a "common soil", but none has delivered sufficient proof to establish its validity as the correct explanation. Indeed, even a metabolic syndrome population consists of a heterogeneous grouping of people having different causes for their biologically defined metabolic syndrome. Thus, not all individuals are insulin-resistant, not all insulin-resistant subjects are glucose-intolerant, not all overweight/obese persons are metabolically abnormal, and lean subjects can develop severe metabolic abnormalities. In epidemiologic studies, three or more subpopulations are usually observed. About one third have fatty liver, considered to be a very crucial component in aggravation of metabolic syndrome towards cardiometabolic syndrome (see later), but this also means that two thirds are not falling into this category. Therefore, it is easily understandable that obtaining a unifying conclusion is largely illusory, at least in the present constellation of our knowledge.

The role of main tissues/organs regulating glycemic and lipidic metabolism is another subject of endless debate. Largely due to technical aspects (clamp technique), skeletal muscle has been increasingly and excessively considered as the initial location of insulin resistance, the latter being considered as one (if not the only) principal common denominator responsible for metabolic syndrome and subsequent type 2 diabetes. This view is partly biased by the fact that it is difficult to investigate deep-lying organs, typically the liver. Techniques for evaluating hepatic insulin resistance, such as stable isotopic methods, are available, but in daily medical practice the diagnosis mostly relies on blood parameters. However, one should bear in mind that the liver is the main organ controlling sugar and lipid metabolism, which is illustrated in its crucial anatomic positioning: it receives products from the digestive system as well as from the periphery of the body, transforms and detoxifies various substances, and delivers glucose, lipids, and inflammatory and hemostatic factors, amongst others, to the rest of the body. It is also closely under the control of the brain as well as an intrinsic nervous network in the liver. To complicate the situation further, recent findings indicate that the tissues involved in metabolic regulation, and consequently in cardiometabolic syndrome, exhibit constant cross-talk. For example, experimentally induced changes in skeletal muscle glucose transport capacity can lead to fat accumulation in the liver, ${ }^{1}$ and the severity of hepatic steatosis is linked to skeletal muscle adiposity; ${ }^{2}$ conversely, the liver sends substances capable of inducing peripheral insulin resistance in skeletal muscle into the bloodstream. For example, it was recently reported that the triglyceride content of skeletal muscle is associated with hepatic but not peripheral resistance. ${ }^{3}$ The same holds true for adipose tissue, which is considered by many to be the crucial player in the initiation of insulin resistance and evolution towards metabolic syndrome. Because of these interplays, arguments for "initial causality" can be found for any of at least five organs, ie, pancreas, liver, adipose, skeletal muscle, and brain. ${ }^{4}$ More recent research has revealed that functional (possibly inherited) abnormalities of microvascular mechanisms can initiate insulin resistance in skeletal muscle or adipose tissue, and that this may function in a bidirectional fashion, eg, in the case of sleep apnea and insulin resistance. ${ }^{5}$

The present review therefore deals mainly with what is known and believed to be the case for the "bulk" of the cardiometabolic syndrome population, and focuses on the particular role of the liver and its links with cardiovascular and metabolic syndrome-related diseases. The accompanying bibliography is, by definition, a subjective but well updated selection where readers can easily find further references for more detailed information.

\section{Crucial role of the liver in cardiometabolic syndrome}

The liver clears, metabolizes, detoxifies, and redistributes the absorbed content of food. Its role in established type 2 diabetes is well demonstrated, ${ }^{6}$ but increasing evidence 
implicates this organ in the very early stages of prediabetes. One major finding of the last decade has been the recognition of a prevalence rate of $30 \%$ for hepatic steatosis in the general population, with an even higher prevalence in obese and elderly populations. ${ }^{7}$ About $15 \%-30 \%$ will progress towards a more severe form, known as nonalcoholic steatohepatitis, where severe inflammation and fibrosis develop, leading eventually to liver cancer. ${ }^{8}$ These various forms of the disease are grouped under the term "nonalcoholic fatty liver disease". Given that the present review deals mainly with early prediabetes, our focus is limited to hepatic steatosis, ie, fatty liver.

\section{Factors inducing hepatic insulin resistance and steatosis}

Many but not all metabolic pathways of glucose and lipid metabolism in the liver are under the control of insulin which regulates hepatic glucose output and lipid synthesis. Therefore, any impairment in hepatic insulin sensitivity is rapidly reflected in glucose homeostasis and triglyceride levels. Today, fatty liver is considered as the "hepatic expression of metabolic syndrome". ${ }^{9}$ Hepatic insulin resistance is severe in fatty liver, regardless of the glycemic tolerance status of patients. $^{10}$

\section{Nutritional factors}

High carbohydrate diets and/or excessive dietary fat leading to exaggerated free fatty acid delivery result in hepatic insulin resistance, ${ }^{11}$ diminished fatty acid oxidation, and de novo lipogenesis with triglyceride accumulation in hepatocytes, ${ }^{12}$ a situation which can be clinically observed either by changes in plasma levels of hepatic enzymes, such as alanine aminotransferase (ALT), aspartate aminotransferase (AST), gamma glutamyl transpeptidase (GGT), and high-sensitivity C-reactive protein, or by echosonography of the liver. Liver fat is possibly a better predictor of multiorgan insulin resistance than visceral fat. ${ }^{13}$ Liver enzyme modifications can be detected very early; ${ }^{14}$ some studies suggest that enzymatic abnormalities are a better indicator of liver insulin resistance than the homeostasis model assessment index, and that subjects with hepatic insulin resistance in the highest quartile of elevated enzymes have a ten-fold increase in risk for developing fatty liver. ${ }^{15}$ High fat also modifies the microbiota in the small intestine, promoting intestinal inflammation and gut permeability ("leaky gut") by damaging the proteins responsible for tightness (zonulin, occludin, gliadin), thereby providing the liver via the portal vein with substances that are not sufficiently or not at all managed by Kupffer cells and hepatocytes. ${ }^{16}$ Dysbiosis or overgrowth of gut bacteria (at least in some families) leads to diminished efficiency of the intestinal barrier and translocation of lipopolysaccharide to the liver, ${ }^{17}$ where it then provokes inflammation and liberation of tumor necrosis factor alpha. ${ }^{18}$ Interestingly, transfer of intestinal microbiota from a mouse fed a high-fat diet to a germ-free animal results in development of fatty liver and hyperglycemia in the receiver. ${ }^{19}$ Analysis of liver fat content in obese patients showed that $28 \%$ came from diet, $58 \%$ from nonesterified fatty acids, and $14 \%$ from de novo lipogenesis. ${ }^{20}$ Monosaccharides induce microbial fermentation and are able to induce hepatic lipogenesis. ${ }^{21}$ Finally, some bile acids, such as deoxycholic acid, also disrupt the intestinal barrier. ${ }^{22}$

Fructose is present as concentrated corn syrup in many sodas, and there is presently a lively debate about its importance in the epidemic of hepatic steatosis, particularly in the US. ${ }^{23}$ Indeed, a high fructose intake leads rapidly to hepatic inflammation, stress on the endoplasmic reticulum, and lipoapoptosis, in addition to de novo lipogenesis. ${ }^{24}$ Moreover, fructose generates uric acid which, via activation of fructokinase, induces lipogenesis and accumulation of triglycerides. ${ }^{25}$

Importantly, however, the liver clears various foodstuffs prone to inducing insulin resistance, such as fructose in high amounts, trans fatty acids, alcohol, and branched-chain amino acids. ${ }^{26}$ These substances interfere with the mitochondria, resulting in excessive lipogenesis. Further mechanisms which can induce hepatic insulin resistance and fatty liver, such as the intermittent hypoxia characteristic of obstructive sleep apnea, ${ }^{27}$ require more investigation.

\section{Non-nutritional factors}

The anatomic position of the liver places this organ in close contact with abdominal fat, a tissue known to be highly lipolytic and to liberate many cytokines, including inflammation factors. An increase in visceral fat, usually as a result of high caloric intake, is therefore a preferential source of substances impacting on the liver.

The nervous system closely controls hepatic metabolism. Therefore, stress is another factor likely to affect the liver via neurotransmitters and glucocorticoids. Stimulation of the hypothalamic-pituitary-adrenal axis by chronic or even mild stress is a favored explanation for the cause of cardiometabolic syndrome. ${ }^{28}$ The stress response is largely mediated by cortisol, a substance to which liver cells are exquisitely sensitive and respond to by rapid development of insulin resistance. ${ }^{29}$ An association between cortisol concentrations measured 
in hair and the metabolic syndrome has been reported. ${ }^{30}$ Although the plasma cortisol concentration is a questionable marker of metabolic syndrome, glucocorticoids aggravate insulin resistance and hypertriglyceridemia in the presence of a high-fat diet. ${ }^{31}$

Sleep disorders induce intense sympathetic activation and have recently been shown to result into development of the cardiometabolic syndrome. ${ }^{32}$ Disordered chronobiology is another recently identified source of metabolic disturbances. ${ }^{33}$

\section{Markers of hepatic insulin resistance/steatosis}

Circulating levels of lipids, in particular triglycerides, are a convenient manner to suspect hepatic insulin resistance, but show no specificity. Early good markers are plasma levels of hepatic enzymes, such as ALT, AST, and GGT. High levels of ALT are linked with the severity of hepatic insulin resistance, ${ }^{34}$ and this relationship appears to be independent of other factors. ${ }^{35,36}$ Interestingly, the association between ALT and insulin resistance seems stronger than with dyslipidemia, ${ }^{37}$ suggesting that ALT might be a good and very early indicator of liver damage, ${ }^{14}$ while AST appears to be more linked to central obesity. ${ }^{38}$ However, ALT is poorly linked to cardiovascular disease, in contrast with GGT. ${ }^{39}$ Although it is a marker of metabolic syndrome, GGT is claimed to be associated more with abdominal obesity, ${ }^{40}$ and possibly linked to the presence of oxidative stress. ${ }^{41}$ However, it should be mentioned that liver failure may be observed in the absence of these enzymatic changes. Some studies suggest that bilirubin, which is inversely related to insulin resistance and oxidative stress, ${ }^{42}$ may be another interesting marker of fatty liver. ${ }^{43,44}$

\section{Mechanisms of hepatic insulin resistance/steatosis}

As stated above, insulin resistance in the liver can be induced by many different factors acting directly in the liver or originating from other tissues, such as fat and skeletal muscle. Free radicals and proinflammatory cytokines, such as interleukin-6, are the most widely known ones. ${ }^{45}$ Oxidative stress, often linked to inflammation, is considered to be a main factor in the pathogenesis of fatty liver. ${ }^{46}$ However, the specificity of oxidative stress markers is questionable, given that this process is common to all forms of insulin resistance. Inflammation plays a major role in fatty liver; ${ }^{47,48}$ it can originate from visceral fat and reach the liver, but can also be generated within the liver as a consequence of triglyceride accumulation and endoplasmic reticulum stress. ${ }^{49}$

The liver contains the largest reservoir of macrophages in the body. Kupffer cells, once activated by xenobiotics, generate inflammation, ${ }^{50}$ and these cells can be proinflammatory or anti-inflammatory, which explains some apparently contradictory data showing protection or otherwise by experimental Kupffer cell depletion against high fructose feeding. ${ }^{51,52}$ Thus, the filtering Kupffer cells can lead to liver inflammation by their reaction to an excess of incoming substrates. Complement C3 is involved in all forms of metabolic disorder and interferes with the coagulation system, so is now proposed as another risk factor for cardiometabolic syndrome. ${ }^{53}$

Although a matter of debate, iron overload is increasingly considered to be an integral part of the pathology of nonalcoholic fatty liver disease. ${ }^{54-57}$ Elevated ferritin levels are associated with the development of metabolic syndrome over 5 years, ${ }^{58}$ and appear to be a good predictor of vascular damage. ${ }^{59}$ A combination of high ferritin and low transferrin saturation is characteristic of prediabetes. ${ }^{60}$ Hepcidin increases with the number of features of metabolic syndrome, ${ }^{61}$ promoting release of cytokines and oxidative stress and leading to apoptosis in macrophages. ${ }^{62}$ Interestingly, a high fat/high fructose diet has recently been shown to induce iron overload via a hepcidin-independent pathway, which occurs before develop of hepatic steatosis and insulin resistance. ${ }^{63}$

\section{Links between hepatic disorders and cardiovascular events}

The inflammatory state of the liver in prediabetic states is expected to impact on the cardiovascular system. There is indeed a well known interplay between insulin resistance, inflammation, obesity, and heart disease. ${ }^{64}$ According to data from the Cremona study, fatty liver is linked to mortality, cancer, and cardiovascular disease, the relationship being explained by insulin resistance. ${ }^{65}$ Insulin-resistant individuals of normal weight have a 2.5 -fold increase in risk for heart failure. ${ }^{66}$ Conversely, it must be noted that up to $80 \%$ of patients with heart failure have liver dysfunction, possibly explained by a disturbed hepatic microcirculation, diminished endotoxin clearance, and increasing cytokine levels. ${ }^{67}$

In this context, nonalcoholic fatty liver is now considered to be a cardiovascular risk factor. ${ }^{68}$ Despite similar myocardial blood flow, it appears that myocardial blood reserve is diminished in subjects with fatty liver, whereby the coronary microcirculation becomes impaired. ${ }^{69}$ There exists a well known (bidirectional?) link between insulin 
resistance and carotid intima thickness, ${ }^{70}$ which is even seen in children with metabolic syndrome. ${ }^{71}$ Subjects with fatty liver show increased carotid intima/media thickness if there is also metabolic syndrome, ${ }^{72}$ but others have found this to be independent of metabolic syndrome. ${ }^{73}$ It was reported that, despite many common features, nonalcoholic fatty liver is more closely linked than alcoholic fatty liver to cardiovascular disease. ${ }^{74}$ Cardiac structure is altered in fatty liver, as reflected in left ventricular thickness, while cardiac metabolism appears intact. ${ }^{75}$ Flow-mediated dilatation was shown to be reduced by $30 \%$ in patients with fatty liver. ${ }^{73}$ Venular blood flow velocity has been reported to be increased in the eye, reflecting microvascular damage to the retina. ${ }^{76}$

Metabolic syndrome and inflammatory mediators, such as interleukin-6, C-reactive protein, and fibrinogen, increase the risk of heart failure by up to $20 \%$ if combined. ${ }^{77}$ GGT has been linked to carotid thickness. ${ }^{78}$ Indeed, GGT is closely linked to the existence of plaques. Patients with a fatty liver index $>60$ show an increased risk of atherosclerosis, which is tentatively explained by incorporation of GGT into plaques, where it might trigger iron-dependent oxidation of lowdensity lipoprotein. ${ }^{79} \mathrm{~A}$ high fructose intake is also linked with an elevated incidence of cardiovascular disease. ${ }^{23}$ There is likely a prominent role for high triglycerides, in that while inert triglycerides are harmless, triglycerides containing saturated fatty acids are harmful. ${ }^{80}$ Excessive secretion of very low-density lipoprotein by the fatty liver increases circulating triglyceride levels, resulting in ectopic fat deposition, notably at perivascular and pericardial locations. ${ }^{81}$

\section{Treatment}

In view of the complex etiology and multiple interconnecting mechanisms involved in hepatic insulin resistance and nonalcoholic fatty liver disease, ${ }^{82,83}$ many therapeutic approaches can be envisaged, the main ones being summarized here. Indeed, targets can either focus on factors inducing hepatic steatosis or intrahepatic mechanisms involved in glucose or lipid metabolism. Most subjects with cardiometabolic syndrome, but not all, ${ }^{84}$ show insulin resistance, which therefore represents a preferential target in the available arsenal of therapeutic measures. The key measure is of course a healthy lifestyle, ie, adequate diet and exercise, with weight loss shown to improve hepatic steatosis, at least in subjects who achieve a weight loss greater than 7\%. Recent research suggests that new notions of nutrition, such as chronobiology, should be integrated into dietary measures. Nevertheless, the extent to which lifestyle measures prevent an evolution which often seems ineluctable is still uncertain and the findings of preventive clinical trials are contradictory. Lifestyle measures are advised in any case, however, whether alone or combined with pharmacologic interventions. Beneficial effects are observed, as expected, but are hampered by practical application, often motivation limitations. ${ }^{85}$ It should also be mentioned that some scientists view insulin resistance, and particularly hepatic insulin resistance, as a protective mechanism against substrate overload, ${ }^{86}$ a notion which is worthy of deeper analysis.

\section{Insulin sensitizers}

Given the importance attributed to insulin resistance in the cardiometabolic syndrome, this defect has been the major target for therapy and, consequently, various types of drugs considered to alleviate insulin resistance have been tested. Indeed, re-establishing insulin signaling in hepatocytes is expected to correct defects in both glycemic and lipid metabolic pathways, although it must be borne in mind that not all of these are controlled by insulin.

\section{Metformin}

The biguanide metformin is the leading antidiabetic treatment worldwide, but has many other effects in addition to reducing fasting hyperglycemia; one of its main actions is to improve insulin sensitivity, and many reports have shown that the liver is the preferential target for this compound ${ }^{87}$ Several mechanistic targets have been identified for metformin in the liver, ${ }^{88}$ including stimulation of the postreceptor protein, IRS-2 ${ }^{89}$ In high-fat feeding models, metformin reduced hepatic glucose production by inhibiting gluconeogenesis ${ }^{90}$ and glucose-6phosphatase, ${ }^{91}$ as well as restoring insulin-sensitive liver blood flow. ${ }^{92}$ As mentioned earlier, fructose is another main nutrient prone to promoting hepatic steatosis when consumed in high amounts, and several studies have demonstrated the capacity of metformin to improve fat accumulation in the liver. Attenuation of lipid peroxidation, ${ }^{93}$ reduction in gene expression of the Toll-like receptor- 4 cascade, and lipid metabolism regulated by plasminogen activator inhibitor type-1, as well as inhibition of intestinal permeability, ${ }^{94}$ reduction in intra-abdominal fat mass, ${ }^{95}$ and activation of AMP-activated protein kinase against interleukin-6-induced insulin resistance, are some mechanisms involved in the action of this drug.

Metformin has shown mixed results in humans, ${ }^{96}$ with an effect superior to that of diet seen in a randomized trial. ${ }^{97}$ Interestingly, a recent study investigated the vascular consequences of treatment with metformin in 
patients with nonalcoholic fatty liver disease, and reported improvement in arterial stiffness in addition to changes in blood metabolic parameters and liver enzymes. ${ }^{98}$ However, the latter effect might also be independent of the hepatic action, given that metformin is a pleiotropic drug with many favorable effects on large and especially small vessels. ${ }^{99}$

\section{Thiazolidinediones}

A more recent class of compounds developed as insulin sensitizers are the thiazolidinediones (TZDs). Pioglitazone, the newest TZD, improves histologic parameters in the liver, although patients gain weight. ${ }^{96}$ Pioglitazone also shows beneficial effects in steatohepatitis. ${ }^{100}$

Limited comparisons of metformin versus TZDs have yielded mixed results, with the TZDs ultimately appearing better when considering liver fat and function. ${ }^{101,102}$ Combining both types of drugs (eg, rosiglitazone + metformin) showed only a transient improvement in hepatic insulin sensitivity but a sustained reduction in ALT. ${ }^{103}$

Here again one should take into consideration the question of surrogate versus final outcome parameters. A recent survey of insulin sensitizers in subjects with nonalcoholic fatty liver disease points to the lack of evidence for beneficial effects of these treatments. ${ }^{104}$ On the other hand, caution is advised with TZDs due to their potential to aggravate cardiovascular parameters.

\section{Statins}

Reducing cholesterol and triglycerides is a logical approach when diet fails. Based on their beneficial effect on cholesterol and their potential to protect against cardiovascular disease, statins are increasingly used in nonalcoholic fatty liver disease. In addition to their effects on blood lipids, these drugs are able to reduce tumor necrosis factor alpha, an inflammatory cytokine. However, although ameliorating surrogate markers, statins are not considered to have a convincing effect in nonalcoholic fatty liver disease. ${ }^{105}$ Moreover, some caution is advised, given that statins are prone to augmenting the risk of diabetes. ${ }^{106}$

\section{Antioxidants and anti-inflammatory drugs}

Although oxidative stress is considered a hallmark of prediabetes and diabetes, there is little evidence that antioxidants (usually vitamin E) have clearcut beneficial effects in clinical terms. Few relevant data are available in nonalcoholic fatty liver disease. A survey by the Cochrane database reported that enzyme changes were observed, but no conclusion could be drawn. ${ }^{107} \mathrm{~A}$ very recent review on this question also reached the same conclusion. ${ }^{108}$

In addition to and in combination with oxidative stress, inflammation is another main cause of the metabolic syndrome. The classical concept states that inflammation originates from adipose tissue due to lipid overload in fat cells. ${ }^{109}$ However, it should be borne in mind that the liver is also an important source of inflammation factors, such as C-reactive protein, and a major reservoir of macrophages. In mice, a high-fat diet first leads to hepatic insulin resistance ${ }^{109}$ and then secondarily to inflammation. ${ }^{110}$ Any anti-inflammatory drug may be potentially useful, but no liver-specific treatment has been yet established. ${ }^{111}$

\section{Prebiotics/probiotics}

One of the most interesting findings in recent times has been the role of intestinal permeability in hepatic steatosis, allowing many toxic factors to reach the liver and cause steatosis. Probiotics can improve this defect, and thus appear to be promising agents in the treatment of nonalcoholic fatty liver disease. ${ }^{12,113}$ In an experimental high-fat model, combined administration of Lactobacillus curvatus and Lactobacillus plantarum suppressed the metabolic abnormalities induced by this diet. ${ }^{114}$

Improving the operative capacity of microbiota by providing prebiotics is another more indirect approach. How these microbiotic changes can impact host tissue metabolism and gene expression has been reviewed recently. ${ }^{115}$ Unfortunately, few data are actually available in this field and further clinical proof is awaited, which will hopefully confirm this promising potential.

\section{Vitamins}

Lack of vitamin D has been independently linked to steatosis in subjects with fatty liver and normal enzyme profiles. ${ }^{116}$ A recent meta-analysis concluded that most subjects with nonalcoholic fatty liver are deficient in vitamin D. ${ }^{117}$ Some studies suggest that administration of high-dose vitamin D might lower the risk of liver steatosis in healthy persons. ${ }^{118}$

At present, it is not possible to draw conclusions about the efficacy of treatment in nonalcoholic fatty liver disease (excluding nonalcoholic steatohepatitis), although various approaches sound promising. Indeed, it should be stressed that most studies are of average quality, and importantly, mid-term or long-term clinical studies are essentially missing in this field. ${ }^{119}$ Moreover, mainly surrogate parameters, such as blood lipids or plasma hepatic enzymes, have been measured, but final clinical outcomes, such as prevention 
or reversal of cardiometabolic syndrome, are lacking, in particular for vascular disorders.

\section{Conclusion}

The anatomic position of the liver confers to this organ a central role in the regulation of nutrient absorption, handling, and redistribution. Consequently, it also has a key role in glucose homeostasis, being able to trap or liberate either stored or newly synthesized glucose, functions which are under the control of many mechanisms and more particularly insulin, to which it is exquisitely sensitive. The quantity and quality of absorbed food closely determines hepatic insulin sensitivity. Visceral fat also directly influences these processes. The liver also exchanges information with peripheral organs, notably skeletal muscle, in an ongoing manner. Excess calories from sugars or lipids, and also from qualitative alimentary components, eg, trans fatty acids and high fructose, directly induce hepatic inflammation and/or oxidative stress and hepatic steatosis. It is now clear that a large proportion of prediabetic individuals have excessive fat accumulation in the liver, a situation which can be detected easily by measuring plasma hepatic enzymes or ultrasound imaging of the liver. Liberation by the liver of inflammatory factors, products of oxidative stress, and triglycerides impacts the vascular system, notably the heart. Therapeutic prevention can be achieved by insulin sensitizers (mainly metformin) and possibly the statins and vitamin D. However, the long-term efficacy of these treatments remains to be demonstrated. The recent recognition of increased intestinal permeability (leaky gut), which enables access of unfavorable gut factors to the liver, is another very promising therapeutic strategy, either by eating pro/prebiotics or by administering treatments specifically dedicated to blocking intestinal permeability.

\section{Disclosure}

The author reports no conflict of interest in this work.

\section{References}

1. Kotani K, Peroni OD, Minokoshi Y, Boss O, Khan BB. GLUT4 glucose transporters deficiency increases hepatic lipid production and peripheral lipid utilization. J Clin Invest. 2004;114:1666-1675.

2. Kitajima Y, Hyogo H, Sumida Y, et al. The severity of nonalcoholic steatohepatitis is associated with substitution of adipose tissue in skeletal muscle. J Gastroenterol Hepatol. April 12, 2013. [Epub ahead of print.]

3. Grunnet LG, Laurila E, Hansson O, et al. The triglyceride content in skeletal muscle is associated with hepatic but not peripheral insulin resistance in elderly twins. J Clin Endocrinol Metab. 2012;97:4571-4577.

4. Raz I. Viewpoints on the way to a consensus session. Diabetes Care. 2009;32 Supp1 2:S149-S150.
5. Wiernsperger N, Nivoit P, Bouskela E. Obstructive sleep apnea and insulin resistance: a role for microcirculation? Clinics. 2006;61:253-266.

6. Loria P, Lonardo A, Anania F. Liver and diabetes. A vicious circle. Hepatol Res. 2013;43:51-64.

7. Bellentani S, Scaglioni F, Marino M, Bedoni G. Epidemiology of nonalcoholic fatty liver disease. Dig Dis. 2010;28:155-161.

8. Levene AP, Goldin RD. The epidemiology, pathogenesis and histopathology of fatty liver disease. Histopathology. 2012;61: 141-152.

9. Medilla-Santillan R, Lopez-Velasquez JA, Chavez-Tapia N, et al. Hepatic manifestations of metabolic syndrome. Diabetes Metab Res Rev. March 7, 2013. [Epub ahead of print.]

10. Ortiz-Lopez C, Lomonaco R, Orsak B, et al. Prevalence of prediabetes and diabetes and metabolic profile of patients with nonalcoholic fatty liver disease (NAFLD). Diabetes Care. 2012;35:873-878.

11. Lottenberg AM, Afonso M, Ferrari Lavrador MS, et al. The role of dietary fatty acids in the pathology of metabolic syndrome. $J$ Nutr Biochem. 2012;23:1027-1040.

12. Utzschneider KM, Kahn SE. The role of insulin resistance in nonalcoholic fatty liver disease. J Clin Endocrinol Metab. 2006;91:4753-4761.

13. Korenblat KM, Fabbrini E, Mohammed BS, Klein S. Liver, muscle, and adipose tissue insulin action is directly related to intrahepatic triglyceride content in obese subjects. Gastroenterology. 2008;134:1369-1375.

14. Andre P, Balkau B, Vol S, et al. Gamma-glutamyltransferase activity and development of the metabolic syndrome (IDF definition) in middleaged men and women. Diabetes Care. 2007;30:2355-2361.

15. Sesti G, Fiorentino TV, Hribal ML, Sciacqua A, Perticone F. Association of hepatic insulin resistance indexes to nonalcoholic fatty liver disease and related biomarkers. Nutr Metab Cardiovasc Dis. February 15, 2013. [Epub ahead of print.]

16. Ding S, Chi MM, Scull BP, et al. High-fat diet: bacteria interactions promote intestinal inflammation which precedes and correlates with obesity and insulin resistance in mouse. PLoS One. 2010;5:e12191.

17. Schnabl B. Linking intestinal homeostasis and liver disease. Curr Opin Gastroenterol. 2013;29:264-270.

18. Ilan Y. Leaky gut and the liver: a role for bacterial translocation in nonalcoholic steatohepatitis. World J Gastroenterol. 2012;18:2609-2618.

19. Le Roy T, Llopis M, Lepage M, et al. Intestinal microbiota determines development of non-alcoholic fatty liver disease in mice. Gut. November 29, 2012. [Epub ahead of print.]

20. Donnelly KL, Smith CI, Schwarzenberg SJ, Jessurun J, Boldt MD, Parks EJ. Sources of fatty acids stored in liver and secreted via lipoproteins in patients with nonalcoholic fatty liver disease. J Clin Invest. 2005;115:1343-1351.

21. Miele L, Marrone G, Lauritano C, et al. Gut-liver axis and microbiota in NAFLD: insight pathophysiology for novel therapeutic target. Curr Pharm Des. 2013;19:5314-5324.

22. Stenman LK, Holma R, Eggert A, Korpela R. A novel mechanism for gut barrier dysfunction by dietary fat: epithelial disruption by hydrophobic bile acids. Am J Physiol Gastrointest Liver Physiol. 2013;304: G227-G234

23. Wiernsperger N, Geloen A, Rapin JR. Fructose and cardiometabolic disorders: the controversy will, and must, continue. Clinics. 2010;65: 729-738.

24. Basaranoglu M, Basaranoglu G, Sabuncu T, Senturk H. Fructose as a key player in the development of fatty liver disease. World J Gastroenterol. 2013;19:1166-1172.

25. Lanaspa MA, Sanchez-Losada LG, Cicherchi C, et al. Uric acid stimulates fructokinase and accelerates fructose metabolism in the development of fatty liver. PLoS One. 2012;7:e47948.

26. Bremer AA, Mietus-Snyder M, Lustig RH. Toward a unifying hypothesis of metabolic syndrome. Pediatrics. 2012;129:557-570.

27. Mirrakhimov AE, Polotsky VY. Obstructive sleep apnea and nonalcoholic fatty liver disease: is the liver another target? Front Neurol. 2012;3:149.

28. Bjorntorp P, Rosmond R. The metabolic syndrome - a neuroendocrine disorder? Br J Nutr. 2000;83:S49-S57. 
29. Anagnostis P, Athyros VG, Tziomalos K, Karagiannis A, Mikhailidis DP. Clinical review: the pathogenetic role of cortisol in the metabolic syndrome: a hypothesis. J Clin Endocrinol Metab. 2009;94: 2692-2701.

30. Stalder T, Kirschbaum C, Alexander N, et al. Cortisol in hair and the metabolic syndrome. J Clin Endocrinol Metab. 2013;98:2573-2580.

31. D'Souza AM, Beaudry JL, Szigiato AA, et al. Consumption of a high-fat diet rapidly exacerbates the development of fatty liver disease that occurs with chronically elevated glucocorticoids. Am J Physiol Gastrointest Liver Physiol. 2012;302:G850-G863.

32. Van Cauter E. Sleep disturbances and insulin resistance. Diabet Med. 2011;28:1455-1462.

33. Garaulet M, Engstroem G, Hedblad B. Chronobiological aspects of nutrition, metabolic syndrome and obesity. Adv Drug Deliv Rev. 2010;62:967-978.

34. Vozarova B, Stefan N, Lindsay RS, et al. High alanine aminotransferase is associated with decreased hepatic insulin sensitivity and predicts the development of type 2 diabetes. Diabetes. 2002;51:1889-1895.

35. Gomez-Samano MA, Cuevas-Ramos D, Mehta R, Brau-Figueroa H, Meza-Arana CE, Gulias-Herrero A. Association of alanine aminotransferase levels (ALT) with the hepatic insulin resistance index (HIRI): a cross-sectional study. BMC Endocr Disord. 2012;12:16.

36. Hanley AJG, Williams K, Festa A, Wagenknecht LE, D’Agnostino RB Jr, Haffner SM. Liver markers and development of the metabolic syndrome. Diabetes. 2005;331:3140-3147.

37. Ghamar-Chehreh ME, Amini M, Khedmat H, et al. Elevated alanine aminotransferase activity is not associated with dyslipidemias, but related to insulin resistance and higher disease grades in non-diabetic non-alcoholic fatty liver disease. Asian Pac J Trop Biomed. 2012;2: $702-706$.

38. Zhang Y, Lu X, Chao M, et al. Positive correlations of liver enzymes with metabolic syndrome including insulin resistance in newly diagnosed type 2 diabetes mellitus. Endocrine. 2010;38:181-187.

39. Ghouri N, Preiss D, Sattar N. Liver enzymes, nonalcoholic fatty liver disease, and incident cardiovascular disease: a narrative review and clinical perspective of prospective data. Hepatology. 2010;52: 1156-1161.

40. Hwang AC, Lin YC, Liu PT, et al. Synergistic effects of gamma glutamyltransferase and obesity on metabolic syndrome, independent of hepatic steatosis. Ann Epidemiol. 2012;32:876-880.

41. Irie M, Sohda T, Iwata K, et al. Levels of the oxidative stress marker gamma-glutamyltranspeptidase at different stages of nonalcoholic fatty liver disease. J Int Med Res. 2012;40:924-933.

42. Lin LY, Kuo HK, Hwang JJ, et al. Serum bilirubin is inversely associated with insulin resistance and metabolic syndrome among children and adolescents. Atherosclerosis. 2009;203:563-568.

43. Kwak MS, Kim D, Chung GE, et al. Serum bilirubin levels are inversely associated with nonalcoholic fatty liver disease. Clin Mol Hepatol. 2012;18:383-390.

44. Jenko-Praznikar Z, Petelin A, Jurdana M, Ziberna L. Serum bilirubin levels are lower in overweight asymptomatic middle-aged adults: an early indicator of metabolic syndrome? Metabolism. 2013;62:976-985.

45. Kumar R, Prakash S, Chhabra S, et al. Association of pro-inflammatory cytokines, adipokines and oxidative stress with insulin resistance and non-alcoholic fatty liver disease. Indian J Med Res. 2012;136: 229-236.

46. Videla LA, Rodrigo R, Araya J, Poniachik J. Insulin resistance and oxidative stress interdependency in non-alcoholic fatty liver disease. Trends Mol Med. 2006;12:555-558.

47. Romeo GR, Lee J, Shoelson SE. Metabolic syndrome, insulin resistance and roles of inflammation- mechanisms and therapeutic targets. Arterioscler Thromb Vasc Biol. 2012;32:1771-1776.

48. Tilg H, Moschen AR. Insulin resistance, inflammation and non-alcoholic fatty liver disease. Trends Endocrinol Metab. 2008;19:371-379.

49. Mollica MP, Lionetti L, Putti R, et al. From chronic overfeeding to hepatic injury: role of endoplasmic reticulum stress and inflammation. Nutr Metab Cardiovasc Dis. 2011;21:222-230.
50. Baffy G. Kupffer cells in non-alcoholic fatty liver disease: the emerging view. J Hepatol. 2009;51:212-223.

51. Lanthier N, Molendi-Coste O, Cani PD, van Rooijen N, Horsmans Y, Leclercq IA. Kupffer cell depletion prevents but has no therapeutic effect on metabolic and inflammatory changes induced by a high-fat diet. FASEB J. 2011;25:4301-4311.

52. Papackova Z, Palenickova E, Dankova H, et al. Kupffer cells ameliorate hepatic insulin resistance induced by high-fat diet rich in monounsaturated fatty acids: the evidence for the involvement of alternatively activated macrophages. Nutr Metab (Lond). 2012;9:22.

53. Hertle E, van Greevenbrock MMJ, Stehouwer CDA. Complement C3: an emerging risk factor in cardiometabolic disease. Diabetologia. 2012;55:881-884

54. Haap M, Machann J, von Friedeburg C, et al. Insulin sensitivity and liver fat: role of iron load. J Clin Endocrinol Metab. 2011;96:E958-E961.

55. Fujita N, Takei Y. Iron overload in nonalcoholic steatohepatitis. $A d v$ Clin Chem. 2011;55:105-132.

56. Nelson JE, Klintworth H, Kowdley KV. Iron metabolism in nonalcoholic fatty liver disease. Curr Gastroenterol Rep. 2012;14:8-16.

57. Shim JJ. Body iron, serum ferritin and nonalcoholic fatty liver disease. Korean J Hepatol. 2012;18:105-107.

58. Park SK, Ryoo JH, Kim MG, Shin JY. Association of serum ferritin and the development of metabolic syndrome in middle-aged Korean men. Diabetes Care. 2012;35:2521-2526.

59. Valenti L, Swinkels DW, Burdick L, et al. Serum ferritin levels are associated with vascular damage in patients with nonalcoholic fatty liver disease. Nutr Metab Cardiovasc Dis. 2011;21:568-575.

60. Cheung CL, Cheung TT, Lam KS, Cheung BM. High ferritin and low transferrin saturation are associated with pre-diabetes among a national representative sample of US adults. Clin Nutr. December 28, 2012. [Epub ahead of print.]

61. Martinelli N, Traglia M, Campostrini N, et al. Increased serum hepcidin levels in subjects with the metabolic syndrome: a population study. PLoS One. 2012;7:e48250.

62. Li JJ, Meng X, Zhang C, et al. Hepcidin destabilizes atherosclerootic plaque via overactivating macrophages after erythrophagocytosis. Arterioscler Thromb Vasc Biol. 2012;32:1158-1166.

63. Tsuchiya H, Ebata Y, Sakabe T, et al. High fat, high fructose diet induces hepatic iron overload via a hepcidin-independent mechanism prior to the onset of liver steatosis and insulin resistance in mice. Metabolism. 2013;62:62-69.

64. Rana JS, Nieuwdorp M, Jukema JW, Kastelein JJ. Cardiovascular metabolic syndrome - an interplay of obesity, inflammation, diabetes and coronary heart disease. Diabetes Obes Metab. 2007;9:218-232.

65. Calori G, Lattuada G, Ragogna F, et al. Fatty liver index and mortality: the Cremona study in the 15th year of follow-up. Hepatology. 2011;54: $145-152$.

66. Voulgari C, Tentolouris N, Dilaveris P, Tousoulis D, Katsilambros N, Stefanadis C. Increased heart failure risk in normal-weight people with metabolic syndrome compared with metabolically healthy obese individuals. J Am Coll Cardiol. 2011;58:1343-1350.

67. Valentová M, von Haehling S, Doehner W, Murín J, Anker SD, Sandek A. Liver dysfunction and its nutritional implications in heart failure. Nutrition. 2013;29:370-378.

68. Bhatia LS, Curzen NP, Calder PC, Byrne CD. Non-alcoholic fatty liver disease: a new and important cardiovascular risk factor? Eur Heart J. 2012;33:1190-1200.

69. Nakamori S, Onihsi K, Nakajima H, et al. Impaired myocardial perfusion reserve in patients with fatty liver disease assessed by quantitative myocardial perfusion magnetic resonance imaging. Circ J. 2012;76:2234-2240.

70. Sciacqua A, Marini MA, Hribal ML, et al. Association of insulin resistance indexes to carotid intima-media thickness. PloS One. 2013;8:e53968.

71. Reinehr T, Wunsch R, de Sousa G, Toschke AM. Relationship between metabolic syndrome definitions for children and adolescents and intimamedia thickness. Atherosclerosis. 2008;199:193-200. 
72. Kim HC, Kim DJ, Huh KB. Association between nonalcoholic fatty liver disease and carotid intima-media thickness according to the presence of metabolic syndrome. Atherosclerosis. 2009;204:521-525.

73. Colak Y, Senates E, Yesil A, et al. Assessment of endothelial function in patients with nonalcoholic fatty liver disease. Endocrine. 2013;43:100-107.

74. Sookoian S, Pirola CJ. Systems biology elucidates common pathogenic mechanisms between nonalcoholic and alcoholic-fatty liver disease. PLoS One. 2013;8:e58895.

75. Hallsworth K, Hoolingsworth KG, Thoma C, et al. Cardiac structure and function are altered in adults with non-alcoholic fatty liver disease. J Hepatol. 2013;58:757-762.

76. Gutfreund S, Izkhakov E, Pokroy R, et al. Retinal blood flow velocity in metabolic syndrome. Graefes Arch Clin Exp Ophthalmol. 2013;251: 1507-1513.

77. Barac A, Wang H, Shara NM, et al. Markers of inflammation, metabolic risk factors and incident heart failure in American Indians: the Strong Heart Study. J Clin Hypertens (Greenwich). 2012;14:13-19.

78. Jung CH, Yu JH, Bae SJ, et al. Serum gamma-glutamyltransferase is associated with arterial stiffness in healthy individuals. Clin Endocrinol. 2011;75:328-334.

79. Kozakova M, Palombo C, Eng MP, et al. Fatty liver index, gammaglutamyltransferase and early carotid plaques. Hepatology. 2012;55 1406-1415.

80. Vanni E, Bugianesi E, Kotronen A, De Minicis S, Yki-Järvinen H, Svegliati-Baroni G. From the metabolic syndrome to NAFLD or vice versa? Dig Liver Dis. 2010;42:320-330.

81. Shimabukuro M, Kozuka C, Taira S, et al. Ectopic fat deposition and global cardiometabolic risk: new paradigm in cardiovascular medicine. J Med Invest. 2013;60:1-14.

82. Ibrahim MA, Kelleni M, Geddaway A. Nonalcoholic fatty liver disease: current and potential therapies. Life Sci. 2012;92:114-118.

83. Musso G, Cassader M, Rosina F, Gambino R. Impact of current treatments on liver disease, glucose metabolism and cardiovascular risk in non-alcoholic fatty liver disease (NAFLD): a systematic review and meta-analysis of randomised trials. Diabetologia. 2012;55: 885-904.

84. Karnchanasorn R, Ou HY, Chuang LM, Chiu KC. Insulin resistance is not necessarily an essential element of metabolic syndrome. Endocrine. 2012;43:92-99.

85. Paredes AH, Torres DM, Harrison SA. Treatment of nonalcoholic fatty liver disease: role of dietary modification and exercise. Clin Liver Dis. 2012;1:116-117.

86. Agius L. High carbohydrate diets induce hepatic insulin resistance to protect the liver from substrate overload. Biochem Pharmacol. 2013;85:306-312.

87. Natali A, Ferrannini E. Effects of metformin and thiazolidinediones on suppression of hepatic glucose production and stimulation of glucose uptake in type 2 diabetes: a systematic review. Diabetologia. 2006;49:434-441

88. Radziuk J, Bailey CJ, Wiernsperger N, Yudkin JS. Metformin and its liver targets in the treatment of type 2 diabetes. Curr Drug Targets Immune Endocr Metabol Disord. 2003;3:151-169.

89. Gunton JE, Delhanty PJD, Takahashi SI, Baxter RC. Metformin rapidly increases insulin receptor activation in human liver and signals preferentially through insulin receptor substrate 2. J Clin Endocrinol Metab. 2003;88:1323-1332.

90. Song S, Andrikopoulos S, Filippis C, Thorburn AW, Khan D, Proietto J. Mechanism of fat-induced hepatic gluconeogenesis: effect of metformin. Am J Physiol Endocrinol Metab. 2001;281:E275-E282.

91. Mithieux G, Guignot L, Bordet JC, Wiernsperger N. Intrahepatic mechanisms underlying the effect of metformin in decreasing basal glucose production in rats fed a high fat diet. Diabetes. 2002;51: 139-143.

92. Pasarin M, Abraldes JG, Rodriguez-Vilarrupla A, et al. Insulin resistance and liver microcirculation in a rat model of early NAFLD. J Hepatol. 2011;55:1095-1102.
93. Srividhya S, Anuradha CV. Metformin improves liver antioxidant potential in rats fed a high fructose diet. Asia Pac J Clin Nutr. 2002;11: 319-322.

94. Spruss A, Kanuri G, Stahl C, Bischoff SG, Bergheim I. Metformin protects against the development of fructose-induced steatosis in mice: role of the intestinal barrier function. Lab Invest. 2012;92: 1020-1032.

95. Baret G, Peyronnet J, Grassi-Kassisse D, Dalmaz Y, Wiernsperger N, Géloën A. Increased intraabdominal adipsoe tissue mass in fructose fed rats: correction by metformin. Exp Clin Endocrinol Diabetes. 2002;110:298-303.

96. Shyangdan D, Clar C, Ghouri N, et al. Insulin sensitizers in the treatment of non-alcoholic fatty liver disease: a systematic review. Health Technol Assess. 2011;15:1-110.

97. Garinis GA, Fruci B, Mazza A. Metformin versus dietary treatment in nonalcoholic hepatic steatosis: a randomized study. Int $J$ Obes. 2010;34:1255-1264.

98. Sofer E, Boaz M, Matas Z, Mashavi M, Shargorodsky M. Treatment with insulin sensitizer metformin improves arterial properties, metabolic parameters and liver function in patients with nonalcoholic fatty liver disease: a randomized, placebo-controlled trial. Metabolism. 2011;60:1278-1284.

99. Wiernsperger NF, Bouskela E. Microcirculation in insulin resistance and diabetes: more than just a complication. Diabetes Metab. 2003; 29 Suppl 6:77-87.

100. Boettcher E, Csako G, Pucino F, Wesley R, Loomba R. Meta-analysis: pioglitazone improves liver histology and fibrosis in patients with non-alcoholic steatohepatitis. Aliment Pharmacol Ther. 2012;35: 66-75.

101. Phielix E, Szendrooedi J, Roden M. The role of metformin and thiazolidinediones in the regulation of hepatic glucose metabolism and its clinical impact. Trends Pharmacol Sci. 2011;32:607-616.

102. Tiikkainen M, Häkkinen AM, Korsheninnikova E, Nyman T, Mäkimattila S, Yki-Järvinen H. Effects of rosiglitazone and metformin on liver fat content, hepatic insulin resistance, insulin clearance and gene expression in adipose tissue in patients with type 2 diabetes. Diabetes. 2004;53:2169-2176.

103. Retnakaran R, Ye C, Hanley AJ, Harris SB, Zinman B. Discordant effects on central obesity, hepatic insulin resistance, and alanine aminotransferase of low-dose metformin and thiazolidinedione combination therapy in patients with impaired glucose tolerance. Diabetes Obes Metab. 2012;14:91-93.

104. Carulli L, Maurantonio M, Hebbard L, Baldelli E, Loria P, George J. Classical and innovative insulin sensitizing drugs for the prevention and treatment of NAFLD. Curr Pharm Des. 2013;19:5280-5296.

105. Lonardo A, Loria P. If steatosis is the atherosclerosis of the liver, are statins the "aspirin" for steatosis? Dig Liver Dis. 2012;44:451-452.

106. Preiss D, Sattar N. Statins and the risk of new-onset diabetes: a review of recent evidence. Curr Opin Lipidol. 2011;22:460-466.

107. Lirussi F, Azzalini L, Orando S, Orlando R, Angelico F. Antioxidant supplements for non alcoholic fatty liver disease and/or steatohepatitis. Cochrane Database Syst Rev. 2007;24:CD004996.

108. Musso G, Anty R, Petta S. Antioxidant therapy and drugs interfering with lipid metabolism: could they be effective in NAFLD patients? Curr Pharm Des. 2013;19:5297-5313.

109. Turner N, Kowalski GM, Leslie SJ, et al. Distinct patterns of tissuespecific lipid accumulation during the induction of insulin resistance in mice by high-fat feeding. Diabetologia. 2013;56:1638-1648.

110. Lee YS, Li P, Huh JY, et al. Inflammation is necessary for long term but not short-term high fat diet-induced insulin resistance. Diabetes. 2011;60:2474-2483.

111. Hirabara SM, Gorjao R, Vinolo MA, Rodrigues AC, Nachbar RT, Curi R. Molecular targets related to inflammation and insulin resistance and potential interventions. J Biomed Biotechnol. 2012;2012:379024.

112. Iacono A, Raso G, Canani RB, et al. Probiotics as an emerging therapeutic strategy to treat NAFLD: focus on molecular and biochemical mechanisms. J Nutr Biochem. 2011;22:699-711. 
113. Vajro P, Mandato C, Veropalumbo C, De Micco I. Probiotics: a possible role in treatment of adult and pediatric non alcoholic fatty liver disease. Ann Hepatol. 2013;12:161-163.

114. Park DY, Ahn YT, Huh CS, et al. Dual probiotic strains suppress high fructose induced metabolic syndrome. World $J$ Gastroenterol. 2013;19:274-283.

115. Delzenne NM, Neyrinck AM, Cani PD. Gut microbiota and metabolic disorders: how prebiotics can work ? Br J Nutr. 2013;109:S81-S85.

116. Barchetta I, Angelico F, Del Ben M, et al. Strong association between non alcoholic fatty liver disease (NAFLD) and low $25(\mathrm{OH})$ vitamin D levels in an adult population with normal serum liver enzymes. $B M C$ Med. 2011;9:85.
117. Eliades M, Spyrou E, Agrawal N, et al. Meta-analysis: vitamin D and non-alcoholic fatty liver disease. Aliment Pharmacol Ther. 2013;38: 246-254.

118. Rhee EJ, Kim MK, Park SE, et al. High serum vitamin D levels reduce the risk for nonalcoholic fatty liver disease in healthy men independent of metabolic syndrome. Endocr J. 2013;60:743-752.

119. Nakajima K. Multidisciplinary pharmacotherapeutic options for nonalcoholic fatty liver disease. Int J Hepatol. 2012;2012:950693.

\section{Publish your work in this journal}

Diabetes, Metabolic Syndrome and Obesity: Targets and Therapy is an international, peer-reviewed open-access journal committed to the rapid publication of the latest laboratory and clinical findings in the fields of diabetes, metabolic syndrome and obesity research. Original research, review, case reports, hypothesis formation, expert opinion and commentaries are all considered for publication. The manuscript management system is completely online and includes a very quick and fair peer-review system, which is all easy to use. Visit http://www.dovepress.com/testimonials.php to read real quotes from published authors.

Submit your manuscript here: http://www.dovepress.com/diabetes-metabolic-syndrome-and-obesity-targets-and-therapy-journal 